\title{
Convergent expansions of the incomplete gamma functions in terms of elementary functions
}

\author{
Blanca Bujanda ${ }^{1}$, José L. López ${ }^{2}$ and Pedro J. Pagola ${ }^{1}$ \\ 1 Dpto. de Ingeniería Matemática e Informática, Universidad Pública de Navarra \\ e-mail: blanca.bujanda@unavarra.es, pedro.pagola@unavarra.es \\ ${ }^{2}$ Dpto. de Ingeniería Matemática e Informática and INAMAT, Universidad Pública de Navarra \\ e-mail: jl.lopez@unavarra.es
}

\begin{abstract}
We consider the incomplete gamma function $\gamma(a, z)$ for $\Re a>0$ and $z \in \mathbb{C}$. We derive several convergent expansions of $z^{-a} \gamma(a, z)$ in terms of exponentials and rational functions of $z$ that hold uniformly in $z$ with $\Re z$ bounded from below. These expansions, multiplied by $e^{z}$, are expansions of $e^{z} z^{-a} \gamma(a, z)$ uniformly convergent in $z$ with $\Re z$ bounded from above. The expansions are accompanied by realistic error bounds.
\end{abstract}

2010 AMS Mathematics Subject Classification: 33B20; 41A58; 41A80.

Keywords \& Phrases: Incomplete gamma functions; convergent expansions; uniform expansions.

\section{Introduction}

The power series expansions of the incomplete gamma functions $\Gamma(a, z)$ and $\gamma(a, z)$ are well known [7, Sec. 8.7, eqs. (8.7.1), (8.7.3)]. For example:

$$
z^{-a} \gamma(a, z)=\sum_{k=0}^{n-1} \frac{(-z)^{k}}{k !(a+k)}+R_{n}^{(0)}(a, z), \quad z \in \mathbb{C}
$$


The Poincaré asymptotic expansions are also well known [7, Sec. 8.11, eq. (8.11.2)]. For example:

$$
z^{-a} \Gamma(a, z)=\frac{e^{-z}}{z}\left[\sum_{k=0}^{n-1} \frac{(1-a)_{k}}{(-z)^{k}}+R_{n}^{(\infty)}(a, z)\right], \quad z \rightarrow \infty .
$$

These expansions have a good property: they are given in terms of elementary functions. But they have an inconvenience: they are not uniformly valid for all values of $z$; the remainder $R_{n}^{(0)}(a, z)$ is unbounded when $|z| \rightarrow \infty$, whereas the the remainder $R_{n}^{(\infty)}(a, z)$ is unbounded when $|z| \rightarrow 0$. Apart from (1), other convergent expansions of the incomplete gamma functions are given for example in [1] and [7, Sec. 8.7] for real z. In [1] we can find expansions given in terms of triple or quadruple sums of elementary functions, whereas in [7, Sec. 8.7] we can find expansions given in terms of Laguerre polynomials $L_{n}^{(a)}(x)$. Uniformity aspects of these expansion are not discussed.

Apart from (2), other asymptotic expansions in terms of elementary and also special functions may be found in [6], [8], [9], [10] and references there in. The expansions derived in these references have a certain uniform character, but not with respect to $|z|$. For example, in [9] we find:

$$
\Gamma(a, x) \sim \frac{1}{2} \Gamma(a) \operatorname{erfc}\left(w \sqrt{\frac{a}{2}}\right)+\Gamma(a) \frac{e^{-a w^{2} / 2}}{\sqrt{2 \pi a}} \sum_{n=0}^{\infty} \frac{c_{n}(w)}{a^{n}}, \quad|a| \rightarrow \infty,
$$

where $\operatorname{erfc}(z)$ is the complementary error function and $w^{2} / 2:=z / a-1-\log (z / a)$. The coefficients $c_{n}(w)$ are elementary functions obtained recursively. In [6] we find expansions like:

$$
\gamma(a, x) \sim z^{a-1 / 2} e^{-z}\left\{\sqrt{\frac{\pi}{2}} e^{u^{2} / 2} \operatorname{erfc}\left(-\frac{u}{\sqrt{2}}\right) \sum_{n=0}^{\infty} \frac{A_{n}(u)}{z^{n / 2}}+\sum_{n=0}^{\infty} \frac{B_{n}(u)}{z^{n / 2}}\right\}, \quad|z| \rightarrow \infty,
$$

valid for $\Re(z-a) \leq 0$ and $|\arg z|<\frac{\pi}{2}$, where $u:=(z-a) / \sqrt{z}$ and the coefficients $A_{n}(u)$ and $B_{n}(u)$ are polynomials in $u$ that may be computed recursively.

As far as we know, none of the known expansions of the incomplete gamma functions satisfy simultaneously the following three properties: (i) it is convergent, (ii) it is given in terms of elementary functions and (iii) it is valid for large and small values of $|z|$. For example, (1) satisfies (i) and (ii), but not (iii); (2) satisfies (ii), but not (i) and (iii); (3) and (4) satisfy none of them. ${ }^{1}$

In this paper we derive expansions of the incomplete gamma functions that satisfy those three properties simultaneously (with $\Re z$ bounded from above or from below). Because of the well known relation $\Gamma(a, z)+\gamma(a, z)=\Gamma(a)$, we only consider here the function $\gamma(a, z)$. The starting point is the integral definition of the incomplete gamma function [7, Sec. 8.2, eq. (8.2.1)]:

$$
\gamma(a, z)=z^{a} \int_{0}^{1} t^{a-1} e^{-z t} d t, \quad \Re a>0 .
$$

\footnotetext{
${ }^{1}$ By no means this fact discredits these expansions, as they are among the most competitive ones to approximate the incomplete gamma functions for large values of their variables.
} 
The power series expansion (1) of $\gamma(a, z)$ may be derived from the above integral by replacing the exponential in the integrand by its Taylor expansion at the origin and interchanging series and integral. The Taylor expansion converges for $t \in[0,1]$, but the convergence is not uniform in $|z|$. Therefore, the resulting expansion (1) is convergent, but not uniformly in $|z|$, because the remainder $R_{n}^{(0)}(a, z)$ is unbounded for large $|z|$.

The asymptotic expansion (2) may be derived from the following integral representation of the incomplete gamma function $\Gamma(a, z)[7$, Sec. 8.6, eq. (8.6.5)]:

$$
\Gamma(a, z)=z^{a} e^{-z} \int_{0}^{\infty}(1+t)^{a-1} e^{-z t} d t, \quad \Re z>0 .
$$

The asymptotic expansions (2) of $\Gamma(a, z)$ follows from Watson's lemma [11, Chap. 1, p. 24, Example 4]. Roughly speaking, Watson's lemma consists of a replacement of the factor $(1+t)^{a-1}$ of the integrand by its Taylor series at the origin and an interchange of the sum and the integral. Now, the situation with respect to the convergence is worse than in the case of the power series expansion of the function $\gamma(a, z)$ mentioned above. The Taylor expansion of $(1+t)^{a-1}$ is not convergent in the whole integration interval, as it is unbounded [11, Chap. 1, p. 24, eq. (5.32)]. This translates into the fact that the expansion (2) that we obtain after interchanging series and integral is not convergent; although it is asymptotic. As the remainder $R_{n}^{(\infty)}(a, z)$ is unbounded for small $|z|$, the expansion is not uniform in $|z|$.

In this paper we propose a different technique that avoids the lack of uniformity in $|z|$ : instead of the Taylor expansion at the origin of the exponential factor in (5), we consider the Taylor expansion of the factor $t^{a-1}$. This factor is not analytic at the origin unless $a \in \mathbb{N}$. In the following section, we consider the Taylor expansion of the function $t^{a-1}$ at any point in the interval $[1 / 2,1]$. The restriction of the base point to this interval is necessary to assure that the integration interval $(0,1)$ is completely contained inside the convergence disk of the Taylor series. We show that the optimal point for a faster convergence is the middle point of the integration interval: $t=1 / 2$; for a different discussion in a similar situation see [2] and [4]. This Taylor expansion is convergent for any $t$ in the integration interval of the integral (5) and, obviously, it is independent of $z$. After the interchange of the series and the integral, this fact, the independence of $z$, translates into a remainder that may be bounded independently of $z$. In order to accelerate the speed of convergence, in Section 3 we consider a faster approximation of the factor $t^{a-1}$ : we divide the integration interval $(0,1)$ into $m$ sub-intervals of equal length and approximate the factor $t^{a-1}$, independently in each interval, by using its Taylor expansion at the middle point of each sub-interval. (Once we have shown in Section 2 that the optimal point for the Taylor expansion in the interval $(0,1)$ is the middle point $1 / 2$, in Section 3 we only consider Taylor expansions at the middle points of the $m$ sub-intervals.) Then, the expansion derived in Section 2 is the particular case $m=1$ of the one derived in Section 3, but we consider first the particular case $m=1$ in the next section for the clarity of the exposition.

As an illustration of the type of approximations that we are going to obtain (see Theorems 1 and 2 below), we derive, for example, the following approximations valid for $\Re z \geq 0$ :

$$
z^{-5 / 2} \gamma\left(\frac{5}{2}, z\right)=\frac{24+12 z-z^{2}}{16 \sqrt{2} z^{3}}-e^{-z} \frac{24+36 z+23 z^{2}}{16 \sqrt{2} z^{3}}+\epsilon(z), \quad|\epsilon(z)| \leq 0.0066
$$




$$
\begin{aligned}
& z^{-5 / 2} \gamma\left(\frac{5}{2}, z\right)=\frac{96+24 z-z^{2}}{64 z^{3}}-\sqrt{3} e^{-z} \frac{32+56 z+37 z^{2}}{64 z^{3}}+ \\
& e^{-z / 2} \frac{32(\sqrt{3}-3)+8(5 \sqrt{3}-9) z+(13 \sqrt{3}-23) z^{2}}{64 z^{3}}+\delta(z), \quad|\delta(z)| \leq 0.0023 .
\end{aligned}
$$

For $z=0$, the right hand side of these equations must be understood in the limit sense.

Section 4 contains some numerical experiments and a few remarks. Through the paper we use the principal argument $\arg z \in(-\pi, \pi]$ for any $z \in \mathbb{C}$ and assume that $\Re a>0$.

\section{A simple uniformly convergent expansion}

The main result of this section is the following theorem.

Theorem 1. For $\Re a>0, z \in \mathbb{C}$ and $n=1,2,3, \ldots$,

$$
z^{-a} \gamma(a, z)=2^{1-a} \sum_{k=0}^{n-1} \frac{(1-a)_{k}}{k !} \gamma_{k}(z)+R_{n}(a, z),
$$

where the functions $\gamma_{k}(z)$ are the elementary functions

$$
\begin{aligned}
\gamma_{k}(z) & :=\int_{0}^{1} e^{-z t}(1-2 t)^{k} d t=\left(1+2 \frac{d}{d z}\right)^{k} \frac{1-e^{-z}}{z}=\frac{(-2)^{k} k !}{z^{k+1}}\left[e_{k}\left(-\frac{z}{2}\right)-e^{-z} e_{k}\left(\frac{z}{2}\right)\right], \\
e_{k}(z) & :=\sum_{j=0}^{k} \frac{z^{j}}{j !}
\end{aligned}
$$

that satisfy the recurrence relation

$$
\gamma_{n+1}(z)=\frac{1+(-1)^{n} e^{-z}}{z}-2 \frac{n+1}{z} \gamma_{n}(z), \quad \gamma_{0}(z)=\frac{1-e^{-z}}{z} .
$$

The remainder is bounded in the form

$$
\left|R_{n}(a, z)\right| \leq H(z) \frac{\left|(1-a)_{n}\right|}{n ! 2^{\Re a-1} \Re a} \max \left\{2^{\Re a-n-1}, 1\right\}
$$

with

$$
H(z):=\left\{\begin{array}{cc}
1 & \text { if } \Re z>0, \\
e^{-\Re z} & \text { if } \Re z \leq 0 .
\end{array}\right.
$$

For $n \geq \Re a-1>0$, the remainder is also bounded in the form

$$
\left|R_{n}(a, z)\right| \leq \frac{2^{1-\Re a}\left|(1-a)_{n}\right|}{(n-1) !(\Re a-1)} \min \left\{\frac{H(z)}{n+1}, \frac{1-e^{-\Re z}}{\Re z}\right\} .
$$


The remainder behaves as $R_{n}(a, z) \sim n^{-\Re a}$ as $n \rightarrow \infty$ uniformly in $z$ with $\Re z \geq \Lambda$, for any fixed $\Lambda \in \mathbb{R}$.

An immediate consequence of this theorem is the following corollary.

Corollary 1. For $\Re a>0, z \in \mathbb{C}$ and $n=1,2,3, \ldots$,

$$
e^{z} z^{-a} \gamma(a, z)=2^{1-a} e^{z} \sum_{k=0}^{n-1} \frac{(1-a)_{k}}{k !} \gamma_{k}(z)+e^{z} R_{n}(a, z)
$$

with $e^{z} R_{n}(a, z)$ bounded by the right hand side of (11), or (13) for $n \geq \Re a-1>0$, with $z$ replaced by $-z$. The expansion (14) is uniformly convergent in $z$ for $\Re z \leq \Lambda$, for any fixed $\Lambda \in \mathbb{R}$.

Proof. of Theorem 1. The derivation of formula (8) follows from the Taylor expansion of the factor $t^{a-1}$ in (5) at the middle point $\lambda=1 / 2$ of the interval $(0,1)$. In principle, and as we have mentioned in Section 1 , instead of the point $\lambda=1 / 2$, we may consider the Taylor expansion of $t^{a-1}$ at any point $\lambda \in[1 / 2,1]$. Then, we first consider any arbitrary point $\lambda \in[1 / 2,1]$, and later we show that the optimal choice is $\lambda=1 / 2$. The Taylor expansion of $t^{a-1}$ at any point $\lambda \in[1 / 2,1]$ is given by the following formula:

$$
t^{a-1}=\lambda^{a-1}\left[\sum_{k=0}^{n-1} \frac{(1-a)_{k}}{k !}\left(1-\frac{t}{\lambda}\right)^{k}+r_{n}(t, a, \lambda)\right], \quad t \in(0,2 \lambda),
$$

where $r_{n}(t, a, \lambda)$ is the Taylor remainder:

$$
r_{n}(t, a, \lambda):=\sum_{k=n}^{\infty} \frac{(1-a)_{k}}{k !}\left(1-\frac{t}{\lambda}\right)^{k}, \quad t \in(0,2 \lambda) .
$$

The restriction $\lambda \in[1 / 2,1]$ guarantees two facts: (i) the point $\lambda$ is included in the interval $[0,1]$ of integration in (5) and (ii) the series (16) is convergent for $t$ in the interval $t \in(0,1)$. After suitable manipulations we obtain

$$
r_{n}(t, a, \lambda)=\frac{(1-a)_{n}}{n !}\left(1-\frac{t}{\lambda}\right)^{n}{ }_{2} F_{1}\left(\begin{array}{c|c}
n+1-a, 1 \\
n+1
\end{array} \mid 1-\frac{t}{\lambda}\right), \quad t \in(0,2 \lambda) .
$$

Replacing $t^{a-1}$ in (5) by the right hand side of (15) and interchanging the sum and the integral we obtain

$$
z^{-a} \gamma(a, z)=\lambda^{a-1} \sum_{k=0}^{n-1} \frac{(1-a)_{k}}{k !} F_{k}(z, \lambda)+\bar{R}_{n}(a, z, \lambda)
$$

with

$$
F_{k}(z, \lambda):=\int_{0}^{1} e^{-z t}\left(1-\frac{t}{\lambda}\right)^{k} d t=\left(1+\frac{1}{\lambda} \frac{d}{d z}\right)^{k} \frac{1-e^{-z}}{z}
$$


and

$$
\bar{R}_{n}(a, z, \lambda):=\lambda^{a-1} \int_{0}^{1} e^{-z t} r_{n}(t, a, \lambda) d t
$$

As $\left|r_{n}(t, a, \lambda)\right|$ is integrable in $(0,1)$ we have that

$$
\left|\bar{R}_{n}(a, z, \lambda)\right| \leq \lambda^{\Re a-1} H(z) \int_{0}^{1}\left|r_{n}(t, a, \lambda)\right| d t,
$$

with $H(z)$ given in (12). From the integral representation of the hypergeometric function [5, Sec. 15.6, eq. (15.6.1)] we have that, for $t \in(0,2 \lambda)$,

$$
\left|{ }_{2} F_{1}\left(\begin{array}{c}
n+1-a, 1 \\
n+1
\end{array} \mid 1-\frac{t}{\lambda}\right)\right| \leq{ }_{2} F_{1}\left(\begin{array}{c}
n+1-\Re a, 1 \\
n+1
\end{array} \mid 1-\frac{t}{\lambda}\right) .
$$

Therefore, from (17) we find that

$$
\begin{aligned}
\left|\bar{R}_{n}(a, z, \lambda)\right| & \leq H(z) \frac{\left|(1-a)_{n}\right|}{n ! \lambda^{1-\Re a}} \int_{0}^{1}\left|1-\frac{t}{\lambda}\right|_{2}^{n} F_{1}\left(\begin{array}{c}
n+1-\Re a, 1 \\
n+1
\end{array} \mid 1-\frac{t}{\lambda}\right) d t \\
& =H(z) \frac{\left|(1-a)_{n}\right|}{n !} G_{n}(a, \lambda),
\end{aligned}
$$

with

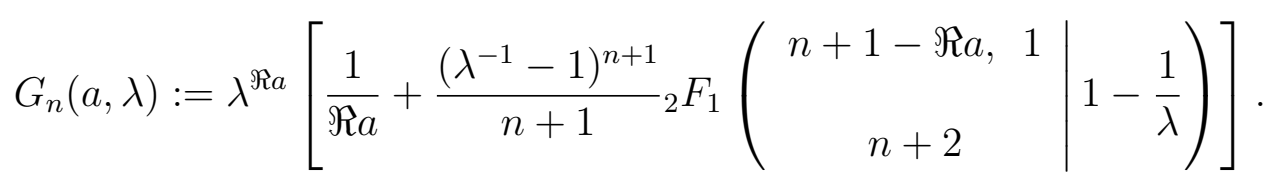

Expansion (18) and bound (21) are valid for any value of $\lambda \in[1 / 2,1]$. We find below the minimum of $G_{n}(a, \lambda)$ for $\lambda \in[1 / 2,1]$ and fixed $n \in N$ and $a$, showing that the value $\lambda=1 / 2$ minimizes the error bound (21). From [5, Sec. 15.5, eq. (15.5.4)] we obtain

$$
\begin{aligned}
\frac{\partial G_{n}(a, \lambda)}{\partial \lambda}= & \lambda^{\Re a-1}\left\{1-\frac{\left(\lambda^{-1}-1\right)^{n}}{n+1}\left[\Re a\left(1-\frac{1}{\lambda}\right){ }_{2} F_{1}\left(\begin{array}{c}
n+1-\Re a, 1 \\
n+2
\end{array} \mid 1-\frac{1}{\lambda}\right)+\right.\right. \\
& \left.\left.+\frac{n+1}{\lambda}{ }_{2} F_{1}\left(\begin{array}{c}
n+1-\Re a, 1 \\
n+1
\end{array} \mid 1-\frac{1}{\lambda}\right)\right]\right\}
\end{aligned}
$$

Now, using the recurrence relation $[5$, Sec. 15.5, eq. (15. 5. 16)] we find

$$
\begin{aligned}
\frac{\partial G_{n}(a, \lambda)}{\partial \lambda}= & \lambda^{\Re a-1}\left[1-\left(\frac{1}{\lambda}-1\right)^{n}{ }_{2} F_{1}\left(\begin{array}{c}
0, n+1-\Re a \\
n+1
\end{array} \mid 1-\frac{1}{\lambda}\right)\right]= \\
& \lambda^{\Re a-1}\left[1-\left(\frac{1}{\lambda}-1\right)^{n}\right] .
\end{aligned}
$$


Therefore $\partial G_{n}(a, \lambda) / \partial \lambda>0$ for all $\lambda \in(1 / 2,1)$. That is, $G_{n}(a, \lambda)$ is a continuous increasing function in $(1 / 2,1)$ and attains its minimum at $\lambda=1 / 2$.

Formulas (8)-(12) of Theorem 1 follow straightforwardly from the previous considerations:

- Formula (8) and the left hand side of (9) are, respectively, formulas (18) and (19) for $\lambda=1 / 2$. The right hand side of (9) follows after a simple computation.

- The recurrence relation (10) follows immediately from (9).

- We have that $R_{n}(a, z)=\bar{R}_{n}(a, z, 1 / 2)$ and then, a bound for $R_{n}(a, z)$ follows from the second line of formula (21) and formula (22) with $\lambda=1 / 2$. Using [5, Sec. 15.5, eq. (15.5.14)] with $a=1, b=n+1-\Re a, c=n+1$ and $z=-1$ we obtain

$$
\left|R_{n}(a, z)\right| \leq H(z) \frac{\left|(1-a)_{n}\right|}{n ! 2^{\Re a-1} \Re a}{ }_{2} F_{1}\left(\begin{array}{c}
n+1-\Re a, 1 \\
n+1
\end{array} \mid-1\right) .
$$

From the integral representation of the hypergeometric function [5, Sec. 6, eq. (15.6.1)] we have that

$$
{ }_{2} F_{1}\left(\begin{array}{c|c}
n+1-\Re a, & 1 \\
n+1 & -1
\end{array}\right)=n \int_{0}^{1}(1-t)^{n-1}(1+t)^{\Re a-n-1} d t \leq \max \left\{2^{\Re a-n-1}, 1\right\} .
$$

Bound (11) follows from (24) and the last inequality.

In order to show (13) we consider the integral representation of the hypergeometric function [5, Sec. 15.6, eq. (15.6.1)] and $n \geq \Re a-1>0$. From that integral representation we have that, for $t \in(0,1)$ :

$$
\left|{ }_{2} F_{1}\left(\begin{array}{c}
n+1-a, 1 \\
n+1
\end{array} \mid 1-2 t\right)\right| \leq n \int_{0}^{1}(1-s)^{n-1}[1-(1-2 t) s]^{\Re a-n-1} d s .
$$

We have that $[1-(1-2 t) s]^{\Re a-n-1} \leq[1-s]^{\Re a-n-1}$ and then

$$
\int_{0}^{1}(1-s)^{n-1}[1-(1-2 t) s]^{\Re a-n-1} d s \leq \int_{0}^{1}(1-s)^{\Re a-2} d s=\frac{1}{\Re a-1} .
$$

Therefore, from (17) we have that

$$
\left|r_{n}\left(t, a, \frac{1}{2}\right)\right| \leq \frac{\left|(1-a)_{n}\right||1-2 t|^{n}}{(n-1) !(\Re a-1)} .
$$

Formula (13) follows from this bound after straightforward computations. Finally, using the Stirling formula and [3, eq. (30)] in (11) or (13) we obtain $R_{n}(a, z) \sim n^{-\Re a}$ as $n \rightarrow \infty$. Any of the bounds (11) or (13) show the uniform character of the expansion for $\Re z>\Lambda$ for any fixed $\Lambda \in \mathbb{R}$. 
We can find a simpler error bound than the ones given in (11) and (13) when the parameter $a$ is real:

Proposition 1. For $a>0, z \in \mathbb{C}$ and $n=1,2,3, \ldots$. The error term $R_{n}(a, z)$ in Theorem 1 is bounded in the form:

$$
\left|R_{n}(a, z)\right| \leq \frac{\left|(1-a)_{n}\right| H(z)}{2^{a-1} a n !}
$$

Proof. We write $p:=\lfloor a\rfloor$, where the symbol $\lfloor a\rfloor$ stands for the biggest integer smaller or equal to $a$, and define $\alpha:=a-p$. Then we have that, for $k \geq p$, that is, $n \geq\lfloor a\rfloor$,

$$
(1-a)_{k}=(-1)^{p}(\alpha)_{p}(1-\alpha)_{k-p} .
$$

Using this equality in (16) we find that

$$
\left|r_{n}\left(t, a, \frac{1}{2}\right)\right| \leq(\alpha)_{p} \sum_{k=n}^{\infty} \frac{(1-\alpha)_{k-p}}{k !}|1-2 t|^{k} .
$$

We introduce this bound in (20) with $\lambda=1 / 2$ and use $\left|e^{-z t}\right| \leq H(z)$ for $t \in[0,1]$. We find (25) after straightforward computations and using (26).

\section{A family of uniformly convergent expansions}

The main result of this section is the following theorem.

Theorem 2. For $\Re a>0, z \in \mathbb{C}$ and $n, m=1,2,3, \ldots$,

$$
z^{-a} \gamma(a, z)=\frac{1}{m} \sum_{k=1}^{m}\left(\frac{2 k-1}{2 m}\right)^{a-1} e^{-(k-1) \frac{z}{m}} \sum_{s=0}^{n-1} \frac{(1-a)_{s}}{s !(2 k-1)^{s}} \gamma_{s}\left(\frac{z}{m}\right)+R_{m, n}(a, z),
$$

where the functions $\gamma_{k}(z)$ are given in (9) and may be computed recursively from (10). On the other hand, for $n \geq \Re a-1>0$, the remainder is bounded in the form

$\left|R_{m, n}(a, z)\right| \leq \frac{\left|(1-a)_{n}\right|}{m^{\Re a} 2^{\Re a-1} n !}\left\{\frac{n}{\Re a-1}+\sum_{k=2}^{m} \frac{e^{-(k-1) \Re z / m}}{(2 k-2)^{n+1-\Re a}}\right\} \times \min \left\{\frac{H(z / m)}{n+1}, \frac{1-e^{-\Re z / m}}{\Re z / m}\right\}$.

with $H(z)$ given in (12). The remainder behaves as $R_{m, n}(a, z) \sim n^{-\Re a}$ as $n \rightarrow \infty$ uniformly in $z$ with $\Re z \geq \Lambda$, for any fixed $\Lambda \in \mathbb{R}$.

An immediate consequence of this theorem is the following corollary.

Corollary 2. For $\Re a>0, z \in \mathbb{C}$ and $m, n=1,2,3, \ldots$,

$$
z^{-a} e^{z} \gamma(a, z)=\frac{1}{m} \sum_{k=1}^{m}\left(\frac{2 k-1}{2 m}\right)^{a-1} e^{(m+1-k) \frac{z}{m}} \sum_{s=0}^{n-1} \frac{(1-a)_{s}}{s !(2 k-1)^{s}} \gamma_{s}\left(\frac{z}{m}\right)+e^{z} R_{m, n}(a, z)
$$


with $e^{z} R_{m, n}(a, z)$ bounded, for $n \geq \Re a-1>0$, by the right hand side of (28) multiplied by $e^{\Re z}$. The expansion (29) is uniformly convergent in $z$ for $\Re z \leq \Lambda$, for any fixed $\Lambda \in \mathbb{R}$.

Proof. of Theorem 2. We divide the integration interval in (5) into $m$ intervals of length $1 / m$ :

$$
z^{-a} \gamma(a, z)=\sum_{k=1}^{m} I_{k}(a, z), \quad \text { with } \quad I_{k}(a, z):=\int_{\frac{k-1}{m}}^{\frac{k}{m}} t^{a-1} e^{-z t} d t
$$

and perform the change of variable $t \rightarrow(t+k-1) / m$ in each one of the integrals $I_{k}(a, z)$ :

$$
I_{k}(a, z)=\frac{e^{-(k-1) \frac{z}{m}}}{m^{a}} \int_{0}^{1}(t+k-1)^{a-1} e^{-\frac{z}{m} t} d t .
$$

We approximate each integral $I_{k}(a, z)$ by replacing the factor $t^{a-1}$ by its Taylor expansion at the middle point of the integration interval. (The experience of the previous section indicates that it is worth to consider only the point $\lambda=1 / 2$ for the Taylor expansion.) Therefore, we have:

$$
(t+k-1)^{a-1}=\left(k-\frac{1}{2}\right)^{a-1}\left\{\sum_{s=0}^{n-1} \frac{(1-a)_{s}}{s !}\left(\frac{1-2 t}{2 k-1}\right)^{s}+r_{n}^{(k)}(t, a)\right\}, \quad t \in(0,1),
$$

where $r_{n}^{(k)}(t, a)$ is the Taylor remainder:

$$
r_{n}^{(k)}(t, a):=\sum_{s=n}^{\infty} \frac{(1-a)_{s}}{s !}\left(\frac{1-2 t}{2 k-1}\right)^{s}=\frac{(1-a)_{n}}{n !}\left(\frac{1-2 t}{2 k-1}\right)^{n}{ }_{2} F_{1}\left(\begin{array}{c|c}
n+1-a, & 1 \\
n+1 & \frac{1-2 t}{2 k-1}
\end{array}\right) .
$$

Replacing (32) into (31) and interchanging the sum and the integral we obtain

$$
I_{k}(a, z)=\frac{1}{m}\left(\frac{2 k-1}{2 m}\right)^{a-1} e^{-(k-1) \frac{z}{m}}\left\{\sum_{s=0}^{n-1} \frac{(1-a)_{s}}{s !(2 k-1)^{s}} \gamma_{s}\left(\frac{z}{m}\right)+R_{n}^{(k)}\left(a, \frac{z}{m}\right)\right\},
$$

with $\gamma_{n}(z)$ given in (9) and

$$
R_{n}^{(k)}(a, z):=\int_{0}^{1} e^{-z t} r_{n}^{(k)}(t, a) d t
$$

From (30) and (34) we find (27) with

$$
R_{m, n}(a, z):=\frac{1}{m} \sum_{k=1}^{m}\left(\frac{2 k-1}{2 m}\right)^{a-1} e^{-(k-1) z / m} R_{n}^{(k)}\left(a, \frac{z}{m}\right) .
$$

In order to derive a bound of $R_{m, n}(a, z)$ we use, on the one hand,

$$
\left|R_{m, n}(a, z)\right| \leq \frac{1}{m} \sum_{k=1}^{m}\left(\frac{2 k-1}{2 m}\right)^{\Re a-1} e^{-(k-1) \Re z / m}\left|R_{n}^{(k)}\left(a, \frac{z}{m}\right)\right| .
$$


And, on the other hand we use that, for $n \geq \Re a-1>0$,

$$
{ }_{2} F_{1}\left(\begin{array}{c|c}
n+1-\Re a, 1 & 1-2 t \\
n+1 & \frac{1-1}{2 k-1}
\end{array}\right) \leq{ }_{2} F_{1}\left(\begin{array}{c|c}
n+1-\Re a, 1 & 1 \\
n+1 & \frac{1}{2 k-1}
\end{array}\right), \quad t \in(0,1) .
$$

From this inequality and equations (33) and (35) we find

$$
\left|R_{n}^{(k)}(a, z)\right| \leq \frac{\left|(1-a)_{n}\right|}{n !(2 k-1)^{n}} F_{1}\left(\begin{array}{c|c}
n+1-\Re a, 1 & 1 \\
n+1 & 2 k-1
\end{array}\right) \int_{0}^{1} e^{-t \Re z}|1-2 t|^{n} d t .
$$

When $k=1$ we have from [5, Sec. 15.4, eq (15.4.20)] that the right hand side of (37) equals $n /(\Re a-1)$. When $k \geq 2$ we have from the integral representation of the hypergeometric function [5, Sec. 15.6, eq. (15.6.1)] that

$$
{ }_{2} F_{1}\left(\begin{array}{c|c}
n+1-\Re a, 1 & 1 \\
n+1 & 2 k-1
\end{array}\right)=n \int_{0}^{1}(1-t)^{n-1}\left[1-\frac{t}{2 k-1}\right]^{\Re a-n-1} d t \leq\left[\frac{2 k-1}{2(k-1)}\right]^{n+1-\Re a} .
$$

When we introduce these bounds in (38) and this in (36) and use either $|1-2 t| \leq 1$ or $e^{-t \Re z} \leq H(z)$ for $t \in[0,1]$ we find $(28)$.

Finally, using the Stirling formula, [3, eq. (30)], [5, Sec. 15.4, eq. (15.4.2)] in (28) we obtain that $R_{m, n}(a, z) \sim n^{-\Re a}$ as $n \rightarrow \infty$. The bound (28) shows the uniform character of the expansion for $\Re z>\Lambda$ for any fixed $\Lambda \in \mathbb{R}$.

We can find a simpler error bound than the one given in (28) when the parameter $a$ is real:

Proposition 2. For $a>0, z \in \mathbb{C}$ and $n=1,2,3, \ldots$ The error term $R_{m, n}(a, z)$ in Theorem 2 is bounded in the form:

$$
\left|R_{m, n}(a, z)\right| \leq \frac{\left|(1-a)_{n}\right| H(z)}{m^{a} 2^{a-1} a n !} \sum_{k=1}^{m}(2 k-1)^{a-n-1} .
$$

Proof. Using equality (26) in (33) we find that

$$
\left|r_{n}^{(k)}(t, a)\right| \leq \frac{(\alpha)_{p}}{(2 k-1)^{n}} \sum_{s=n}^{\infty} \frac{(1-\alpha)_{s-p}}{s !}|1-2 t|^{s} .
$$

We introduce this bound in (35) and use $\left|e^{-z t}\right| \leq H(z)$ for $t \in[0,1]$ to find

$$
\left|R_{n}^{(k)}(a, z)\right| \leq \frac{\left|(1-a)_{n}\right| H(z)}{a n !(2 k-1)^{n}} .
$$

Bound (39) follows by introducing the latter bound in (36), using $H(z / m) e^{-(k-1) \Re z / m} \leq H(z)$ for $1 \leq k \leq m=1,2,3, \ldots$ and after straightforward computations. 


\section{Final remarks and numerical experiments}

Formula (6) is derived immediately from formulas (8) and (11) for $a=5 / 2$ and $n=3$. Formula (7) is derived immediately from formulas (27) and (28) for $a=5 / 2, m=2$ and $n=3$.

From the special values of the incomplete gamma function given in [7, Sec. 8.4, eq. (8.4.7)], it may be deduced that, for integer values of $a=1,2,3, \ldots,{ }^{2}$

$$
z^{-a} \gamma(a, z)=\left(-\frac{d}{d z}\right)^{a-1} \frac{1-e^{-z}}{z}
$$

On the other hand, from (8) and the second equality in (9) we find that, for $\Re a>0$,

$$
z^{-a} \gamma(a, z)=2^{1-a} \sum_{n=0}^{\infty} \frac{(1-a)_{n}}{n !}\left(1+2 \frac{d}{d z}\right)^{n} \frac{1-e^{-z}}{z} .
$$

Using the formal expansion

$$
\left(-\frac{d}{d z}\right)^{a-1}=2^{1-a}\left[1-\left(1+2 \frac{d}{d z}\right)\right]^{a-1}=2^{1-a} \sum_{n=0}^{\infty} \frac{(1-a)_{n}}{n !}\left(1+2 \frac{d}{d z}\right)^{n}
$$

we find that formula (40) is formally valid for $\Re a>0$.

We observe from the bounds given in Theorems 1 and 2 that the approximations given in these theorems may be not very accurate for small values of $\Re a$, specially when $\Re a \in(0,1)$. Then, the smaller $\Re a$ is, the more terms we need in the expansions (8) or (27) to obtain a given accuracy. But the recursion (10) that provides the terms $\gamma_{k}(z)$ of these expansions is not stable for large $z$ and $n$. Then, for numerical purposes, when $\Re a$ is small we suggest to use first the recursion [7, Sec 8.8, eq. 8.8.1] $a \gamma(a, z)=\gamma(a+1, z)+z^{a} e^{-z}$ and then the recursion (10) for large $\Re a$ and a not very large number of terms $n$.

The functions $\gamma_{k}(z)$ defined in (9) are defined for any $z \in \mathbb{C}$; although for $z=0$, the right hand side of the first line in (9) must be understood in the limit sense. In particular, for $z=0$, the right hand side of formulas (6) and (7) must be understood in the limit sense. Near $z=0$, the right hand side of formulas (6) and (7), and of formulas (8), (14), (27) and (29) in general, are differences of large numbers. Then, for numerical purposes, when $z$ is close to zero, it is convenient to approximate the right hand side of these formulas by their Taylor approximation at $z=0$.

The presence of the exponential factor in the function $H(z)$ for $\Re z>0$ suggests that it is more appropriate to use the expansions given in theorems 1 and 2 for positive values of $\Re z$ as well as the expansions given in corollaries 1 and 2 for negative values of $\Re z$.

The numerical experiments given in figures 1 and 2 show the accuracy of expansions (8) and (27). From Figure 2 we see that, the larger we take the order $m$ of expansion (27), the

\footnotetext{
${ }^{2}$ This equality also follows from (5) for $a=1,2,3, \ldots$ replacing $t^{a-1} e^{-z t}$ by $(-d / d z)^{a-1} e^{-z t}$.
} 

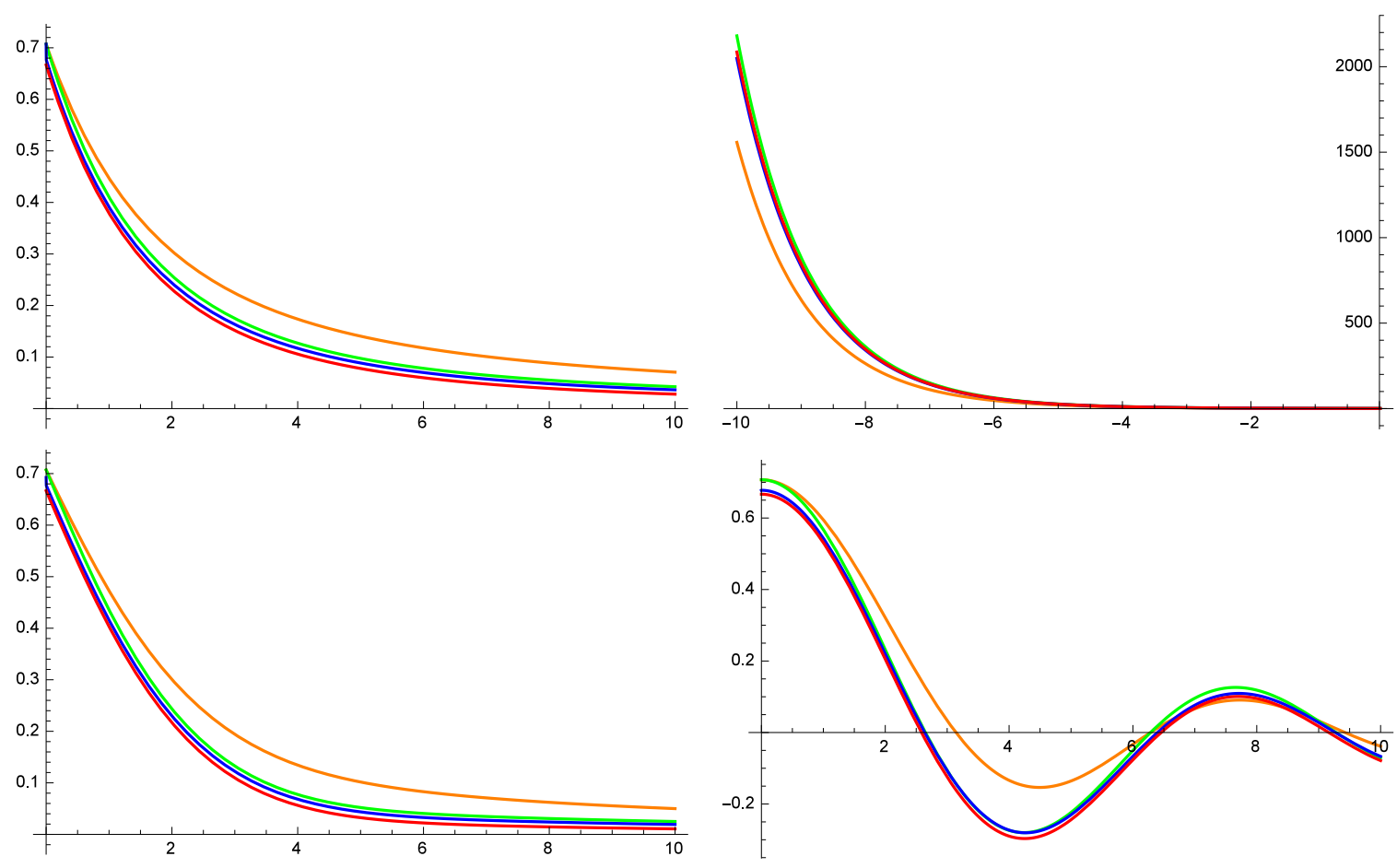

Figure 1: Graphics of $\gamma_{3 / 2}(z)$ (red) and the approximations given in Theorem 1 for $n=1$ (orange), $n=2$ (green), $n=3$ (blue) in several intervals: [0,10] (top left), $[-10,0]$ (top right), $\left[0,10 e^{i \pi / 4}\right]$ (bottom left) and $[0,10 i]$ (bottom right). The bottom graphics correspond to the real part, the pictures for the imaginary part are similar.

more accurate the approximation is. This is just what we should expect from a theoretical point of view, as the larger $m$ is, the more uniform the approximation of the factor $t^{a-1}$ is in the whole integration interval $[0,1]$. On the other hand, the smaller $m$ is, the simpler the expression (27) is.

Expansions (8), (14), (27) and (29) have the three important properties mentioned in the introduction: (i) they are convergent, (ii) they are given in terms of elementary functions and (iii) they are uniformly valid in $z$ (with $\Re z$ bounded from below or from above). As far as we know, other expansions of the incomplete gamma functions given in the literature, in particular the ones given in the introduction, do not satisfy the three properties simultaneously. In Figures 3 and 4 we show some numerical experiments to compare the accuracy of expansions (8) and (27) and the accuracy of other known expansions of similar analytical complexity like (1) and (2). Expansion (1) is more competitive for small $|z|$, expansion (2) is more competitive for large $|z|$ and expansions (8) and (27) are more competitive for intermediate values of $|z|$ and are uniform in $|z|$ (with $\Re z$ bounded from below or from above). 

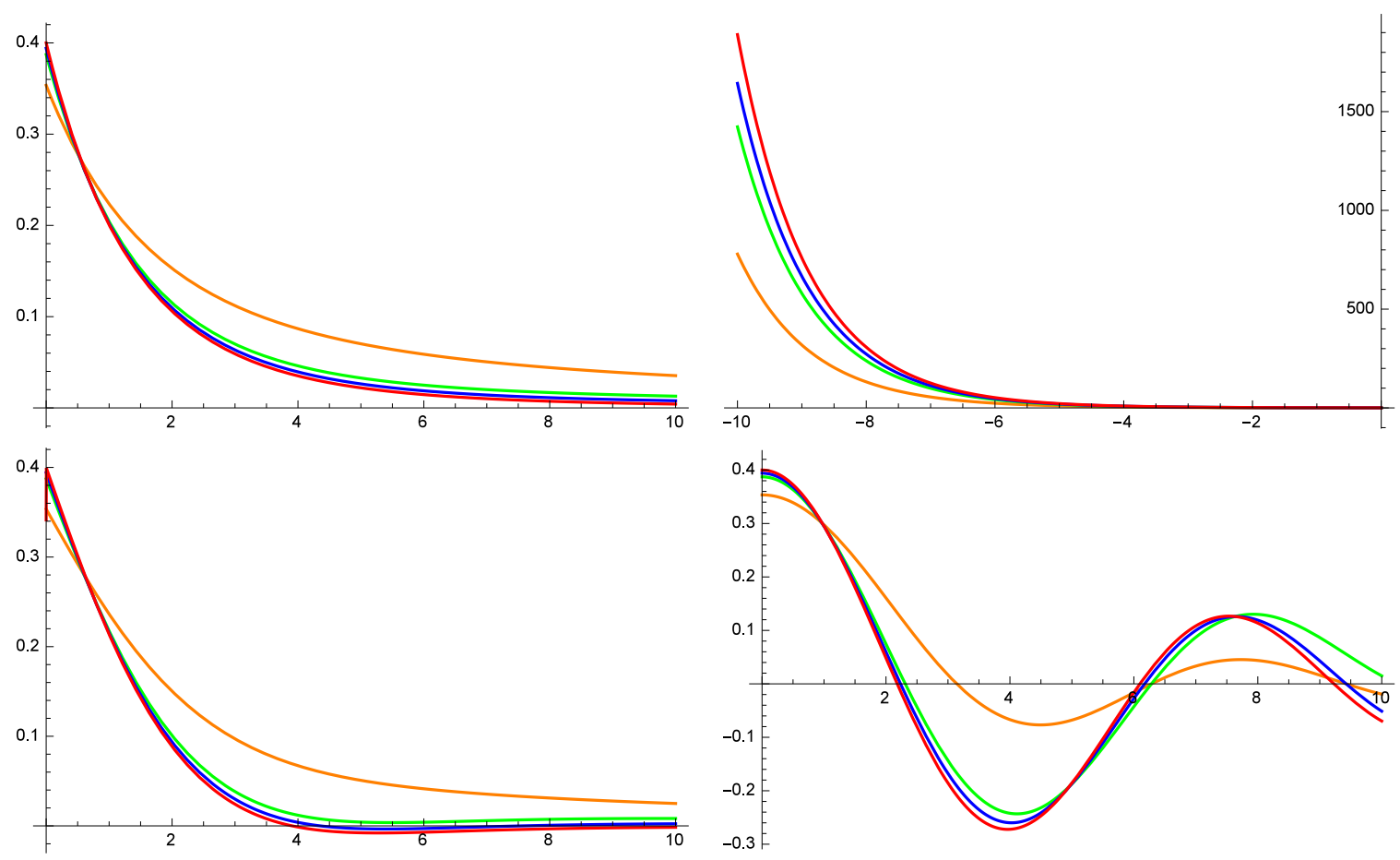

Figure 2: Graphics of $\gamma_{5 / 2}(z)$ (red) and the approximations given in Theorem 2 for $n=1$ and $m=1$ (orange), $m=2$ (green), $m=3$ (blue) in several intervals: [0,10] (top left), [-10,0] (top right), $\left[0,10 e^{i \pi / 4}\right]$ (bottom left) and $[0,10 i]$ (bottom right). The bottom graphics correspond to the real part, the pictures for the imaginary part are similar. 

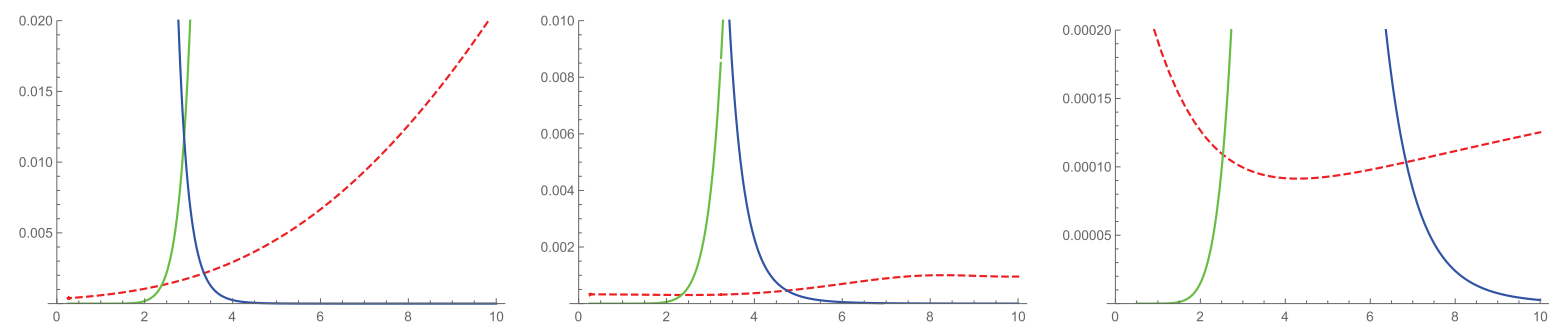

Figure 3: Absolute value of the relative errors in the approximation of $\gamma_{5 / 2}(z)$ by using the uniform expansion (8) (red and dashed), the Taylor expansion (1) (green) and the asymptotic expansion (2) (blue) in the intervals $z \in[0,10]$ (left), $z \in\left[0,10 e^{i \pi / 4}\right]$ (middle) and $z \in\left[0,10 e^{i \pi}\right]$ (right). We have taken $n=10$.
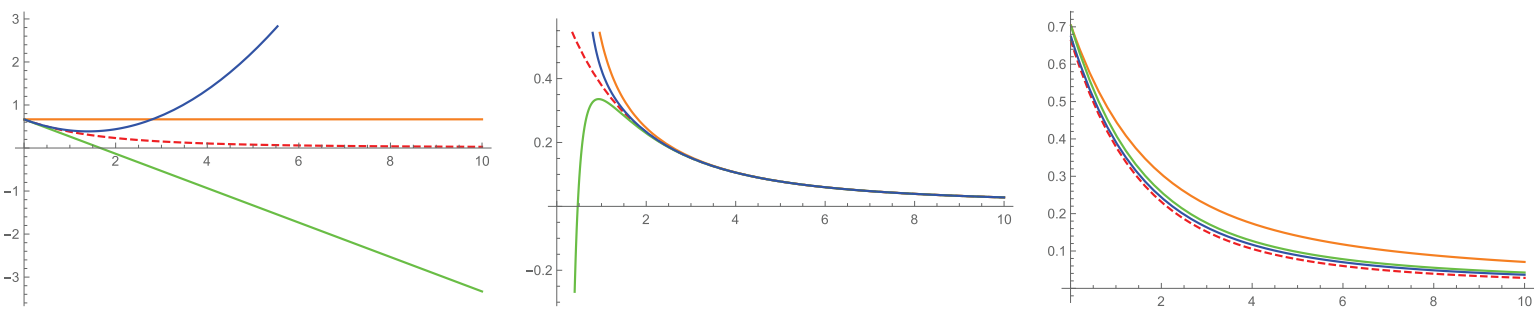

Figure 4: Graphics of $\gamma_{3 / 2}(z)$ (red and dashed) and the approximations (1) (left), (2) (middle) and (8) (right), for $n=1$ (orange), $n=2$ (green), $n=3$ (blue) in the interval $z \in[0,10]$. The pictures for complex $z$ are similar.

\section{Acknowledgments}

This research was supported by the Spanish Ministry of Economía y Competitividad, projects MTM2014-53178-P and TEC2013-45585-C2-1-R. The Universidad Pública de Navarra is acknowledged by its financial support. The referees are acknowledged by their comments and improving suggestions.

\section{References}

[1] P. Amore, Asymptotic and exact series representations for the incomplete Gamma function, Europhys. Lett., 71 n. 1 (2005) 1-7.

[2] J. L. Lopez and N. M. Temme, New series expansions of the Gauss hypergeometric function, Adv. Comput. Math., 39 n. 2 (2013) 349-365.

[3] J.L. López, P.J. Pagola and E. Pérez Sinusía, A simplification of Laplace's method: Applications to the Gamma function and the Gauss hypergeometric function, J. Approx. Theory, 161 (2009), 280-291. 
[4] J. L. Lopez and E. Perez-Sinusia, New Series Expansions for the Confluent Hypergeometric Function M(a,b,z), Appl. Math. Comput., 235 (2014) 26-31.

[5] A. B. Olde Daalhuis, Hypergeometric Function, in: NIST Handbook of Mathematical Functions, Cambridge University Press, Cambridge, 2010, pp. 383-402 (Chapter 15).

[6] R. B. Paris, A uniform asymptotic expansion for the incomplete gamma function, J. Comput. Appl. Math., 148 (2002) 323-339.

[7] R. B. Paris, Incomplete Gamma Functions, in: NIST Handbook of Mathematical Functions, Cambridge University Press, Cambridge, 2010, pp. 173-192 (Chapter 8).

[8] N. M. Temme, Uniform asymptotic expansions of the incomplete gamma functions and the incomplete beta function, Math. Comput., 29 n. 132 (1975) 1109-1114.

[9] N. M. Temme, The asymptotic expansion of the incomplete gamma functions, SIAM J. Math. Anal., 10 (1979) $757-766$.

[10] N. M. Temme, Uniform asymptotics for the incomplete gamma functions starting from negative values of the parameters, Methods Appl. Anal., 3 n. 3 (1996) 335-344.

[11] R. Wong, Asymptotic approximations of integrals, Academic Press, New York, 1989. 